\title{
Light-Activated Porphyrinoid-Capped Nanoparticles for Gas Sensing
}

Gabriele Magna $^{1 \dagger}$, Mounika Muduganti ${ }^{2 \dagger}$, Manuela Stefanelli ${ }^{1}$, Yuvaraj Sivalingam ${ }^{3}$, Francesca Zurlo ${ }^{1}$, Elisabetta Di Bartolomeo ${ }^{1}$, Alexandro Catini ${ }^{2}$, Eugenio Martinelli ${ }^{2}$, Roberto Paolesse $^{1}$, Corrado Di Natale ${ }^{2 *}$

${ }^{\dagger}$ these authors contributed equally

*corresponding author

dinatale@uniroma2.it

1. Department of Chemical Science and Technologies, University of Rome Tor Vergata. Via della Ricerca Scientifica, 00133 Roma, Italy

2. Department of Electronic Engineering, University of Rome Tor Vergata, Via del Politecnico 1, 00133 Roma, Italy

3. Department of Physics and Nanotechnology, SRM Institute of Science and Technology, Kattankulatur, Tamilnadu-603203, India

\section{Keywords}

Porphyrins; Corroles; ZnO nanoparticles; Gas sensor array; Spherical Principal Component Analysis

\footnotetext{
Abstract

The coupling of semiconductors with organic molecules results in a class of sensors whose chemoresistive properties are dictated by the nature of dyes. Organic molecules generally reduce conductivity, but in the case of optically active dyes, such as porphyrinoids, the conductivity is restored by the illumination with visible light. In this paper, we investigated the gas sensing properties of $\mathrm{ZnO}$ nanoparticles coated with porphyrins and corroles. Under light illumination, the resistance of these materials increases with the adsorption of volatile compounds but decreases when these are strong electron donors.

The behavior of these sensors can be explicated on the basis of the structural difference between free-base porphyrin and corrole, the influence of coordinated metal and the corresponding electronic structures.
} 
These sensors are promising electronic noses that combines the selectivity to strong electron donors with the broad-selectivity towards the other classes of chemicals. An efficient representation of the data of this peculiar array can be obtained by replacing the Euclidean distance with the angular distance. To this end, a recently introduced spherical principal component analysis algorithm is applied for the first time to gas sensor array data. Results show that a minimal gas sensor array (four elements) can produce a sort of chemotopic map which enables to cluster a very large class of pure chemical vapors. Furthermore, this map provides information about the composition of complex odor matrices, such as the headspaces of beef meat and their evolution over the time.

\section{Introduction}

The broad functional spectrum of organic chemistry allows the preparation of countless materials with predictable affinity and selectivity for volatile compounds. ${ }^{1}$ The sensitivity of these materials is generally based on sorption mechanisms and they can be specifically synthesized in order to modulate the intensity of the different interactions with airborne molecules, such as Van der Waals forces, hydrogen bond, $\mathrm{CH}-\pi, \pi-\pi$ interactions, and coordination bonds. ${ }^{2}{ }^{3}$ Among the measurable quantities affected by the interactions, electric resistance is surely the most appealing for the simplicity of the device fabrication. Furthermore, the low-complexity of the related electronics ensures the miniaturization of sensor arrays in integrated chips. ${ }^{4}$ However, only few organic materials are conductive enough to allow the fabrication of chemoresistors.

Indeed, several interesting materials, such as porphyrins, are not among these materials. Thus, they are used in combination with either mass or optical transducers. ${ }^{5}$ For instance, arrays of porphyrin coated quartz microbalances have been demonstrated to be selective and sensitive enough to identify diseases from the analysis of breath, as shown in the case of lung cancer ${ }^{6}$ and tuberculosis. ${ }^{7}$ A great advance in terms of sensor system design could be achieved if the sensitivity and selectivity of porphyrins were transferred to resistive sensors.

Several approaches have been proposed to design conductive sensors based on non-conductive sensitive materials. In these sensors conductive and non-conductive elements are combined so that the absorption of molecules in the non-conductive part modulates the current flowing in the conductive counterpart. Examples of such sensors include organically coated carbon nanotubes ${ }^{8}$ or silicon nanowires. ${ }^{9}$ In these devices the adsorption of polar molecules has a similar effect to gate voltage in field effect transistors. ${ }^{10} 11$ Alternative approaches us composite materials made of blends of a non-conductive, but chemically sensitive polymer, and carbon 
black microparticles. ${ }^{12}$ A suitable balance of the two components results in carbon black particles that are close enough to activate charges transfer among them. A more controlled situation is obtained with covalently coated metal nanoparticles. ${ }^{13}{ }^{14}$ In these materials, the thickness of the organic layer, and subsequentially the intergrain current, can be finely tuned. In all these cases, the absorption of airborne molecules modulates the barrier between the conductive particles, changing the overall resistance.

A peculiar combination of organic-inorganic hybrids is represented by wide band gap semiconductors coated with organic-dyes in which the chemical sensitivity along with conductivity is usually activated by providing energy through an external source. For example, temperature has been utilized to prompt the sensitivity of porphyrins coated $\mathrm{ZnO}^{15}$ and photoactivation with ultraviolet light has been proposed as a practical alternative to temperature to promote the sensitivity of nanostructured metal oxide semiconductors. ${ }^{16} 17$

However, the behavior of porphyrins and $\mathrm{ZnO}$ hybrids suggests that the sensitivity of these materials can be easily activated by visible light. Indeed, such hybrid materials were studied in depth for their photoconversion properties and they are the core of organic sensitized solar cells. ${ }^{18}$ Thus, the starting action of the photovoltaic process, i.e. the electron injection into the metal oxide conduction band operated by the dye, can also be exploited for sensing using porphyrins that are excellent materials for both solar cells and sensors. 195

Following this idea, we studied the properties of porphyrin coated $\mathrm{ZnO}$ nanostructures and found that exposure to visible light not only increases the conductivity, due to the charge injection from porphyrins to $\mathrm{ZnO}$, but also significantly alters sensitivity towards analytes. We demonstrated this effect in the case of ethanol and triethylamine, as representative of two classes of volatile compounds, showing, in particular, that the sensitivity is promoted towards amine. ${ }^{20}$ We have further investigated the sensitivity of porphyrin functionalized $\mathrm{ZnO}$ nanoparticles comparing triethylamine with a restricted number of volatile compounds. ${ }^{21}$ These preliminary works were limited to only a few analytes and to porphyrin derivatives. In this work the potentialities of photoactivated resistive sensors are fully explored, demonstrating the scope of this approach using two different classes of porphyrinoids to functionalize $\mathrm{ZnO}$ nanoparticles, and investigating the response towards a wide class of volatile compounds and real samples.

In particular, we investigated the possibility of modulating the rich properties of porphyrins by synthetic modifications, which can allow to optimize and differentiate their sensitive mechanism, mimicking what occurs in Nature, where porphyrins and chlorins play completely different functions essential for life by subtle variations of the molecular skeleton. We chose 
corroles that are cognate molecules of porphyrins, sharing the molecular skeleton of corrin, the nucleus of Vitamin B12, with two directly linked pyrrole rings, but retaining an aromatic character like porphyrins. ${ }^{22}$ This results in a more electron-rich contracted system with respect to porphyrin that has assumed a prominent position in the porphyrinoid field, showing peculiar coordination and optical properties, with high luminescence yields. ${ }^{23}$ This different behavior with respect to porphyrins has opened the way for the application of corrole derivatives in catalysis and medicine. ${ }^{24} 25$ More recently, we also demonstrated the exploitation of copper corroles in perovskite based photovoltaic cells. ${ }^{26}$ All these results make corroles interesting for the target sensing application.

In this paper we investigated the role of the organic layer comparing the performance of freebase and copper complexes of tetraphenylporphyrin and triphenylcorrole.

Results show that exposure to electron donors increases conductivity, which instead decreases when exposed to other volatile molecules. However, cross-sensitivity, typical of porphyrinoids, is still preserved and it enables the use of arrays of these sensors as an electronic nose.

Due to the ambipolar character of sensor responses, data points span a large portion of the sensor space. Proper analysis of these data can be obtained considering, rather than the usual Euclidean distance, the angular distance using the spherical Principal Component Analysis (PCA). ${ }^{27}$ This is a recently introduced algorithm which is used for the first time here to process gas sensor array data. Spherical PCA emphasizes the identification of volatile compounds regardless of their concentration and provides a representation of sensor array responses analogous to the olfactory chemotopic map. This map can be used as a sort of atlas to infer the composition of unknown samples and to represent the evolution over time of complex samples such as the process of spoiling in beef meat.

\section{Materials and methods}

Reagents and solvents were purchased from either Sigma Aldrich, Merck, or Carlo Erba and were used as received.

Thin-layer chromatography (TLC) was performed on Merck silica gel plates. Chromatographic purification on column was accomplished by using silica gel 60 (70230 mesh, Sigma Aldrich) as the stationary phase. ${ }^{1} \mathrm{H}$ NMR spectra of the porphyrinoid derivatives were recorded with a Bruker AV300 spectrometer $(300 \mathrm{MHz})$ in $\mathrm{CDCl}_{3}$ and were internally referenced to residual proton solvent signals $\left(\mathrm{CDCl}_{3} ; \delta=7.26 \mathrm{ppm}\right)$. A FAB mass spectrum was obtained with a VG-Quattro spectrometer in the positive-ion 
mode by using $\mathrm{CHCl}_{3}$ as the solvent and m-nitrobenzyl alcohol (Sigma Aldrich) as the matrix. UV/Vis spectra were recorded with a Cary 100 spectrophotometer.

A microstructural and chemical analysis of the hybrid nanoparticles $\mathbf{H}_{2}$ TPP, $\mathbf{H}_{3}$ TPC, CuTPP and CuTPC was carried out by using a Scanning Electron Microscope (SEM, SUPRA 35, Carl Zeiss SMT, Oberkochen, Germany), equipped with energy-dispersive X-ray microanalysis (EDX, INCAx-sight, model: 7426, Oxford Instruments, Abingdon, Oxfordshire, U.K.) and Transmission Electron Microscopy (TEM, JEM-2100 Plus, JEOL Japan). A gold interdigitated pair of electrodes was purchased from DropSens (Metrohm DropSens InterDigitated Electrodes Ref. G-IDEAU5, bands/gaps dimension: $5 \mu \mathrm{m}$, Number of digits: $250 \times 2$, DropSens, Oviedo Spain). The ultrasonic bath was performed utilizing FB 15047 purchased from Fisher Scientific (FB 15047, 37 kHz, 3090W). Sensor resistances were acquired by remote controlling a Keithley 2001 equipped with a TCSCAN card utilizing a GPIB card; data were automatically collected on a PC by ad hoc MATLAB script. The white LED utilized was 1 W High Power LED Light Source, ASMT-AW00, Broadcom Limited, San Jose, CA, USA. The vapor dilutions were controlled by a couple of mass flow controllers (MKS Instruments, Andover, MA, USA).

\section{Preparation of porphyrin and corrole derivatives}

The target monocarboxylic porphyrin and corrole free-bases (НгрTPP and Нзр TPC) were the result of hydrolysing in alkaline conditions the corresponding methyl esters Н2рТРPMе and НзрТРСМе whose preparation followed different synthetic strategies. As far as porphyrin is concerned, we used a mixed aldehyde condensation in a 10:1 proportion with 10 equivalents of pyrrole in boiling acetic acid was used to achieve the $A_{3} B$-porphyin isomer in good yield, ${ }^{28}$ while the analogous corrole derivative was prepared by the acidcatalyzed condensation of 5-phenyldipyrromethane and 4-acetossibenzaldehyde according to the $\mathrm{H}_{2} \mathrm{O} / \mathrm{MeOH}$ method. ${ }^{29}$ The related copper complexes (CupTPP and CupTPC) were prepared by following standard metalation protocols used for these macrocycles. Schemes and reaction conditions describing all the synthetic steps are thoroughly reported in Supporting Information (Scheme S1 and Scheme S2).

\section{Synthesis of hybrid ZnO nanoparticles}


The procedure utilized to synthesize porphyrin capped nanoparticles is schematically illustrated in Figure 1. Hybrid nanoparticles were prepared with a one-pot method, where porphyrinoids and zinc acetate dihydrate, acting as $\mathrm{ZnO}$ precursors, were mixed together and the material was grown with a hydrothermal procedure. ${ }^{30}$ The organic molecules utilized as dyes were 5-(4'-Carboxyphenyl)-10,15,20-triphenylporphyrin (H2pTPP) and 10-(4'Carboxyphenyl)- 5,15-triphenylcorrole (НзрTPC) and their corresponding copper complexes (CupTPP and CupTPC). All molecules were functionalized with a carboxylic group to promote the interaction with $\mathrm{ZnO}^{31} 32$

Towards this aim, $5.3 \mathrm{~g}$ of zinc acetate dihydrate, as precursor for $\mathrm{ZnO}$ crystals growth, and $5.7 \mathrm{mg}$ of respective organic macrocycles were dissolved in $80 \mathrm{~mL}$ of ethanol, and the resulting solution was sealed in an autoclave reactor. The synthesis was performed by placing the reactor in an oven at $120^{\circ} \mathrm{C}$ for $12 \mathrm{~h}$. In the end, hybrid nanoparticles are formed appearing as a colored suspension. Nanoparticles were collected upon the subsequent cleaning procedure in ethanol and finally the samples were dried at room temperature to obtain powders. The hydrothermal synthesis procedure and the other treatments are totally compatible with the porphyrinoids.

\section{Sensors preparation}

To deposit hybrid materials onto the chemoresistive sensors, $100 \mathrm{mg}$ of hybrid nanoparticles powder were suspended in $4 \mathrm{~mL}$ of toluene. An ultrasonic bath was utilized to promote the dispersion and suspension of nanoparticles. Five drops of $100 \mu \mathrm{L}$ of homogeneous solution were spotted onto each interdigitated electrode to obtain a uniform thick layer. 


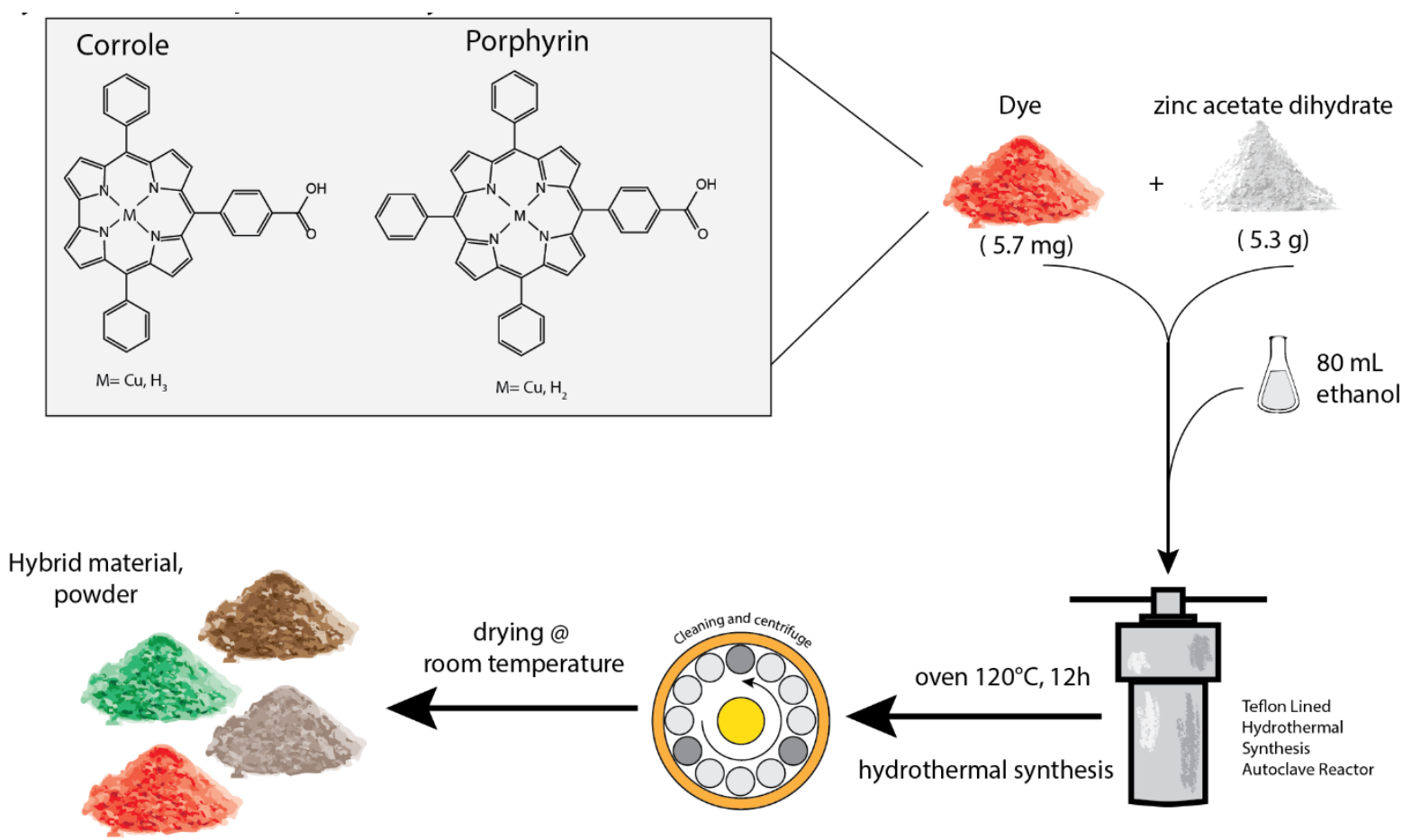

\section{Sensor fabrication}

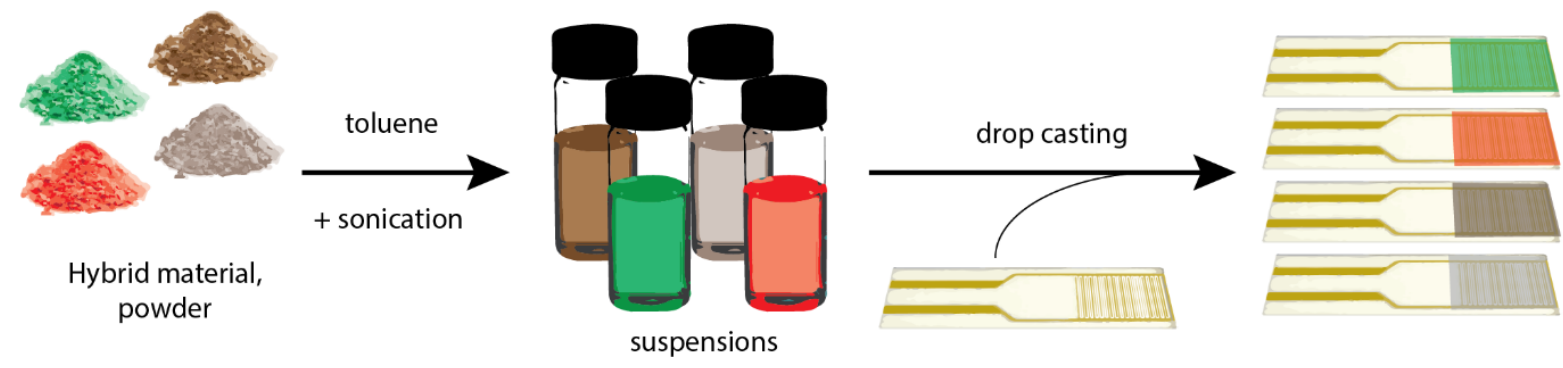

Figure 1: Material synthesis and sensor preparation.

\section{Sensors characterization}

Sensors were accommodated in a sealed chamber provided with a transparent glass window allowing for illumination with a white LED. Sensors were exposed to vapors of the following pure compounds: butanol, ethanol, water, hexane, styrene, toluene, tetrahydrofuran, nitromethane, acetone, aniline, triethyl phosphite, triethylamine, ethylenediamine, allylamine, 1-butylamine. These compounds were chosen as representative of different chemical families. Different vapor concentrations were obtained mixing saturated vapors with a stream of synthetic air. The mixture was controlled by a couple of mass flow controllers. Saturated pressures were calculated with Antoine's law using the parameters available at the NIST database (https://webbook.nist.gov/chemistry). 
Dilution percentages were in the range of $1-25 \%$ and the total flux was always maintained at $200 \mathrm{sccm}$. The stream of synthetic air was also used to restore the sensor response and to define the sensors baseline. Except when exposed to water vapor, all measurements were performed in an anhydrous gas stream.

The electric resistance of the sensors was continuously measured with a multichannel multimeter connected to a computer, where the data were continuously stored.

Sensors were also tested on real samples from the headspace of meat. Minced and sliced beef was bought in a local market. In all cases $2.5 \mathrm{~g}$ of meat was dried with cellulose paper and put in a vial. In the case of minced meat, samples were stored in a refrigerator at $4^{\circ} \mathrm{C}$ and taken out every half-hour. In the case of sliced meat, samples were prepared the first day and placed in a deep freezer $\left(-18^{\circ} \mathrm{C}\right)$ to prevent degradation. At 12 hour intervals, the vials were moved to refrigerators $\left(4^{\circ} \mathrm{C}\right)$ to allow spoilage. Twelve samples were stored in a refrigerator over a sixdays period. On the last day, all the vials were measured following a randomized order.

Each vial was kept at laboratory temperature $\left(25 \pm 2^{\circ} \mathrm{C}\right)$; for $30 \mathrm{~min}$. before being measured. The headspace was sampled by a 200 sccm of synthetic air stream into the sensors chamber.

\section{Data analysis}

All data were analyzed in Matlab (R_2020A) using the Statistics and Machine Learning Toolbox (version 11.7). Spherical PCA was calculated with a Matlab script developed by the MInDS@Mines lab led by Dr. Hua Wang at the Colorado School of Mines (http://minds.mines.edu/publication/2019sdm_spca/).

\section{Results and discussion}

\section{Material characterization}

After the synthesis, since each porphyrinoid is characterized by a distinct visible spectrum, the nanoparticles show a peculiar color depending on the organic molecules utilized as a coating dye. However, in the UV-Vis spectra the thinness of the dyes and the light scattering from the nanoparticles prevent the emergence of the porphyrinoids spectral pattern from the baseline (see Figure S1 in the Supporting Information). However, the color of nanoparticles can be properly observed when they are suspended at high concentration in a proper solvent (see Figure S2 in the Supporting Information). 
SEM investigation evidences that nanoparticles are heterodispersed and form well packed films once spotted onto substrates, as shown in Figure 2A.

Figure 3 shows the TEM images, taken at two magnifications, of the four porphyrinoid coated $\mathrm{ZnO}$ nanoparticles. Particles possess $20 \mathrm{~nm}$ average dimensions and most do not show the typical hexagonal pyramidal structures, usually observed in pure $\mathrm{ZnO}$ nanoparticles ${ }^{33}$. Their shape and size are strongly influenced by the presence of porphyrinoids during the growth of crystals. High magnification images show that, with respect to porphyrins, the addition of corrole to the nanoparticle precursor results in the formation of larger particles with a rounded shape. Furthermore, even at higher magnification, the presence of porphyrinoids is not evident, most likely because of the thickness of the layer. To confirm the presence of dyes, elemental analysis was performed by SEM (Figure 2B). The balance between $\mathrm{Zn}$ and $\mathrm{O}$ contents rules out the presence of high degree defects in the metal oxide lattice. Carbon content is proportional to the amount of dyes covering the nanoparticles. Remarkably, and even considering the limited accuracy of the quantitative analysis with EDX, the amount of dyes seems to be correlated to the presence of $\mathrm{Cu}$ metal in the aromatic ring of dyes. So we can surmise that hybrid materials made of CupTPP and CupTPC contain more organic molecules compared to the materials made with $\mathbf{H}_{2}$ PTPP and $\mathbf{H}_{3}$ PTPC.
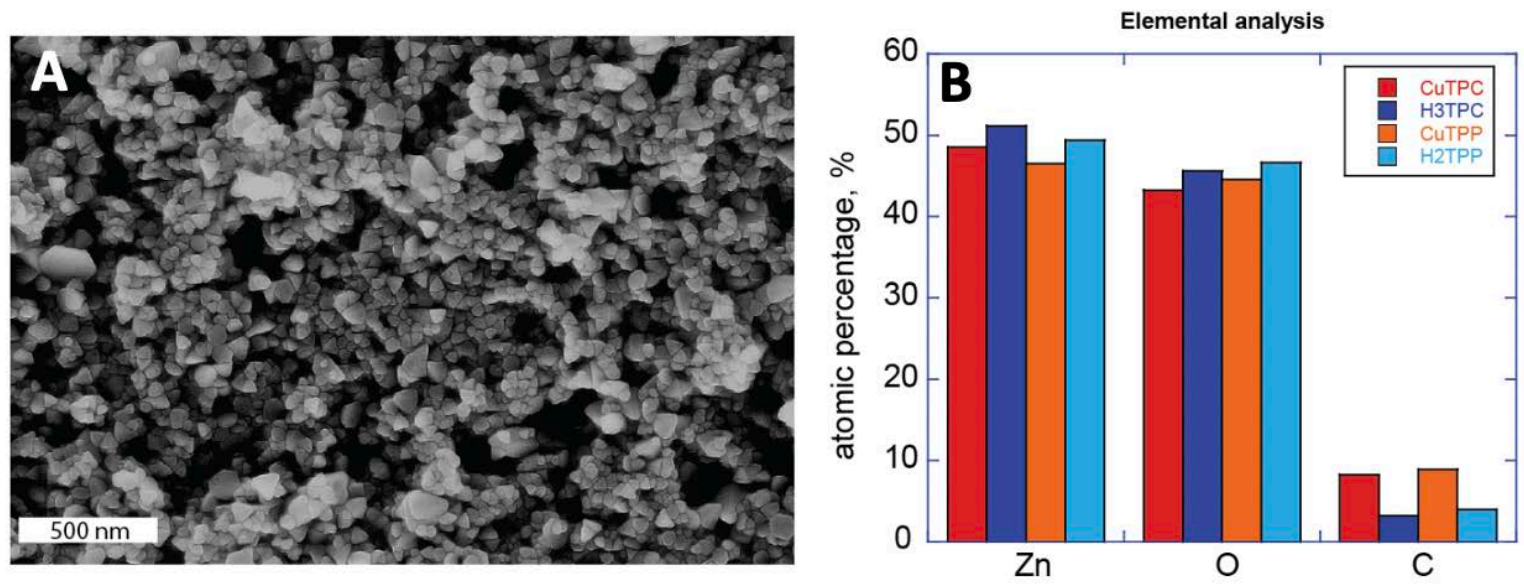

Figure 2. A) SEM micrography of hybrid ZnO particles filmed on the sensor substrate. B) EDX elemental analysis. Atomic ratio of Zn, oxygen, and Carbon measured in films of porphyrinoids coated ZnO nanoparticles. 


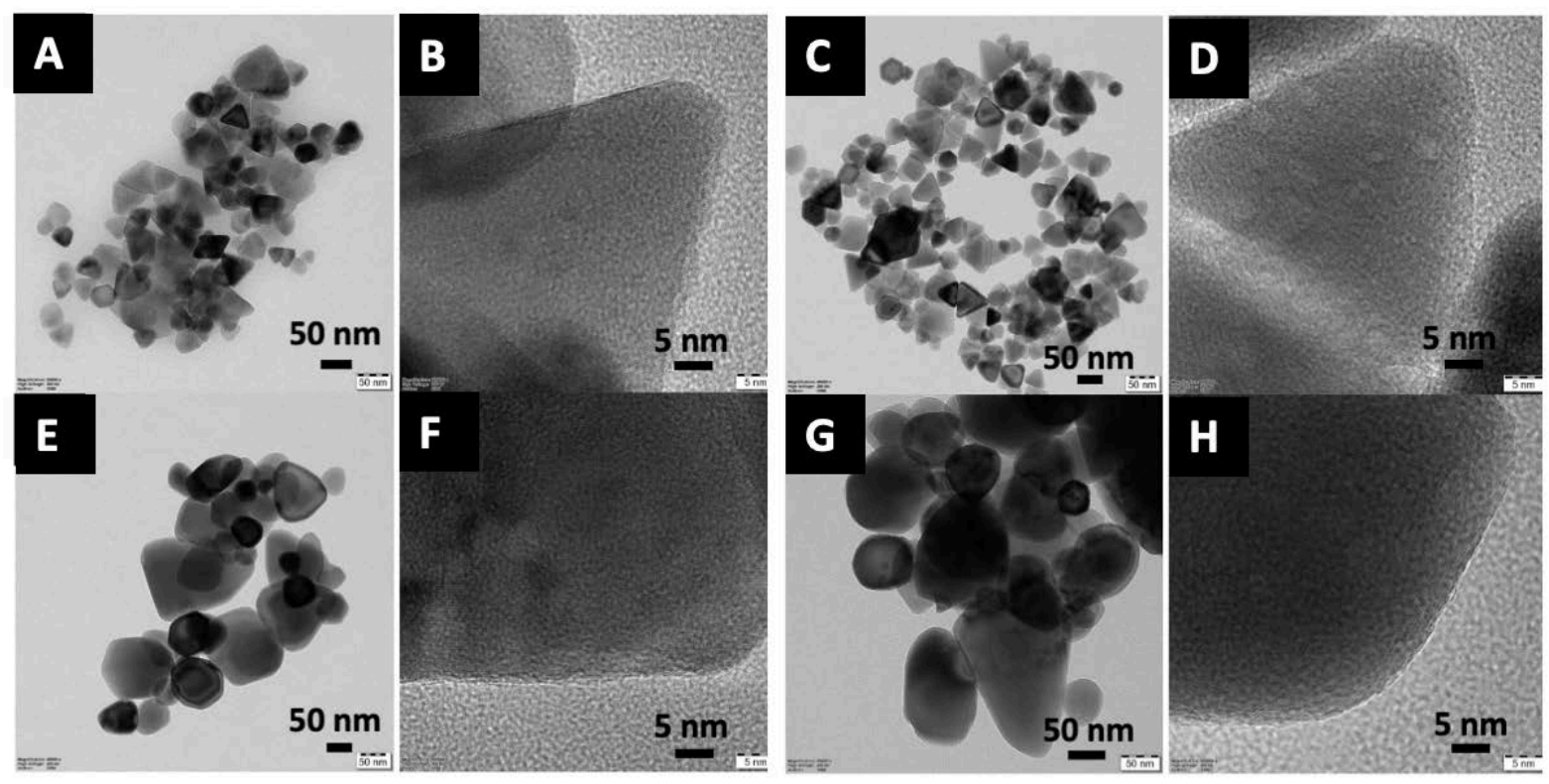

Figure 3: TEM images at two resolutions. A,B: $H_{2}$ TPP; C,D: CuTPP; E,F: $H_{3} T P C ; G, H$ : CUTPC

\section{Gas sensitivity}

Gas sensitivity was measured exposing the sensors to variable concentrations of the 15 volatile compounds.

During the measurements, sensors were constantly kept under the illumination of a white LED. A photodiode was used to ensure that, except for short time fluctuations, the intensity of light was constant.

The normalized change of sensor resistance under the white LED illumination is shown in figure S3 in the Supporting Information. The resistance of sensors under illumination and in a stream of synthetic air is $11 \mathrm{M} \Omega$ and $18 \mathrm{M} \Omega$ for $\mathbf{H}_{2} \mathbf{T P P}$ and $\mathbf{H}_{3} \mathbf{T P C}$, the corresponding metal complexes show a larger resistance of the order of $51 \mathrm{M} \Omega$ and $158 \mathrm{M} \Omega$ for CuTPP and CuTPC respectively. The response times to an abrupt illumination are $232 \mathrm{~s}$ and $281 \mathrm{~s}$ for $\mathbf{H}_{3}$ TPC and CuTPC; and 153 s and 256 s for $\mathbf{H}_{2}$ TPP and CuTPP respectively.

Free-base compounds coated nanoparticles show a shorter response time and lower resistance than the corresponding metallated macrocycles. As suggested by elemental analysis, the percentage of metal complexes is larger with respect to the free-bases, this should result in a larger gap between nanoparticles that is responsible for the larger resistance and response time to illumination. 
Sample concentrations are obtained by diluting the saturated vapors, then the difference in the saturation pressures of the different volatile compounds changes the concentration ranges. The sensors' response to exposure to volatile compounds was expressed by relative variation of the sensor resistance defined as the ratio between the variation of the resistance measured before and at the end of the exposure to the volatile compound normalized by the resistance before the exposure. The relationship between sensor responses and volatile compound concentrations is well fitted by a linear equation for all cases and in the investigated range (see Figure S4 in the Supporting Information).

The adsorption of volatile compounds gives rise to a general increase in resistance. This can be explained considering that the adsorbed molecules form an additional barrier for the electrons moving among adjacent nanoparticles. An important exception to this behavior occurs when a charge transfer takes place between the adsorbed molecule and the porphyrinoids.

Among the investigated volatile compounds, amines and triethyl phosphite represent good ligands in coordination chemistry. However, this interaction is additional with respect to Van der Waals, $\pi-\pi$, and hydrogen bonds. The balance between these interactions is variable and it depends, apart from on the nature of the molecules, on the tridimensional arrangement of porphyrinoids around the nanoparticles too. Only the adsorption of triethylamine and triethyl posphite results in a decrease of resistance. In the case of 1-butylamine, the decrease of resistance is observed in sensors coated with free-base macrocycles ( $\mathbf{H}_{2} \mathbf{p}$ TPP and $\mathbf{H}_{3} \mathbf{p}$ TPC) and only НзTPC decreases its resistance when it is exposed to allylamine. Finally, ethylenediamine vapors increase the resistance of all sensors. 

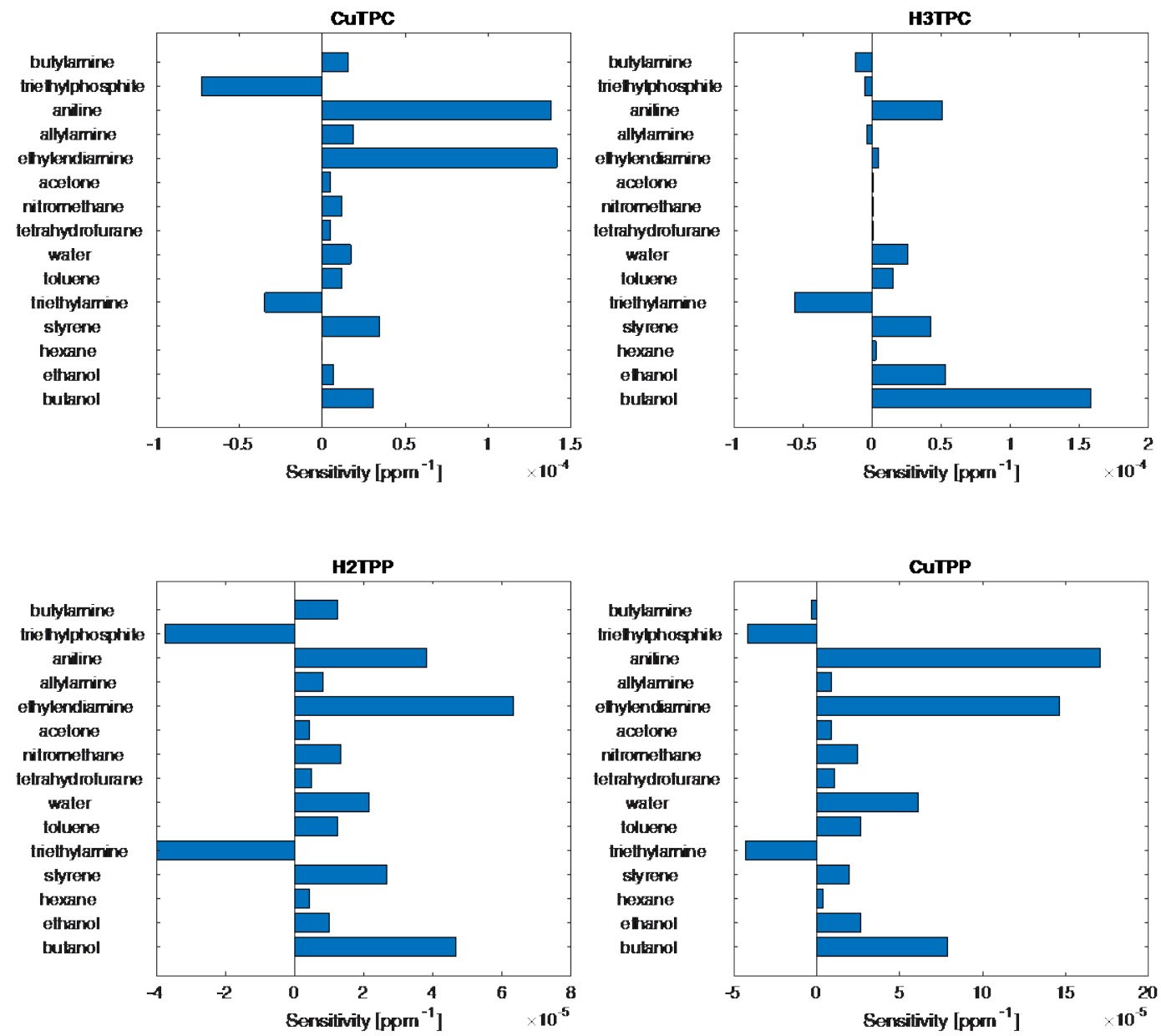

Figure 4: Sensitivity of the four sensors respect to the investigated volatile compounds.

In the investigated range of concentrations, all sensors' data are adequately fitted by a linear relationship with the concentration. In this case, the slope of the linear function is the sensitivity of the sensor. ${ }^{34}$

For each volatile compound only a limited range of concentration was investigated, the smallest concentration of each compound corresponding to about $1 \%$ of its saturation pressure. Due to the narrow interval of concentration, the validity of the extension of the measured sensitivity to the whole range of concentrations and, in particular, to the low concentrations, cannot be straightforwardly assumed, but neds to be experimentally verified.

In Figure 4 show the pattern of sensitivity for each sensor. It is important to observe that all sensors are almost equally sensitive to the set of volatile compounds, and the differences, when they exist, are not easily captured by a simple comparison of the sensitivity patterns. 
However, a scrutiny of sensitivities provides an insight into the characteristics of sensors. The intrinsic difference between porphyrins and corroles is appreciable considering the sensitivity patterns of $\mathbf{H}_{2}$ TPP and $\mathbf{H}_{3}$ TPC. The structural difference of the free-bases can explicate the response pattern observed. The additional hydrogen atom in the corrole pocket leads to a nonplanar structure that increases both the macrocycle acidity and the ability to form hydrogen bonds ${ }^{35}$, thus favoring interaction with alcohols and bases.

The presence of the copper atom tends to smooth the sensor's behavior, introducing axial coordination among possible interactions, but again some important differences can still be observed, due to the macrocycle character. Although in both macrocycles copper has the same oxidation state, the trianionic and electron-rich nature of corrole leads to a ligand-to-metal electron transfer, with the macrocycle oxidation to a cation radical. This feature leads to a facile reduction of copper corroles, making it more sensitive to donor ligands. ${ }^{23}$ Furthermore, the metal also interferes with the molecular arrangement around the nanoparticles affecting the efficiency of Van der Waals forces.
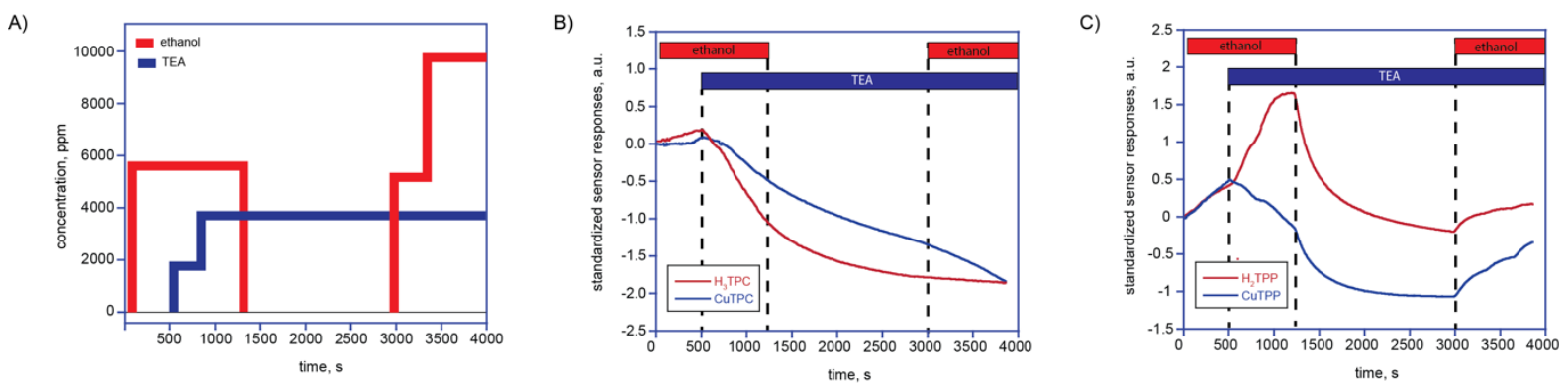

Figure 5: Response of sensors to variable mixtures of ethanol and triethylamine. A) Ethanol and triethylamine concentration sequence. B) Signals of corroles functionalized sensors. C) Signals of porphyrins functionalized sensors.

To investigate the interference between interaction mechanisms, sensors were exposed to variable mixtures and sequences of ethanol and triethylamine, chosen among the compounds that elicit an opposite sign of the sensor's response. Figure 5 shows triethylamine and ethanol concentrations and the corresponding sensors signals.

The concentration of triethylamine was changed in presence of an ethanol background and vice versa (Figure 5A). The first exposure to ethanol in a background of synthetic air increases the resistance of all sensors. For corrole-based sensors (Figure 5B), the following exposure to triethylamine decreases the resistance and, in presence of triethylamine, the sensor becomes insensitive to ethanol. A different behavior is observed in porphyrin-based sensors (Figure 5C). 
CuTPP shows contemporary sensitivity to both compounds. Resistance decreases when triethylamine is added to an ethanol background and increases when ethanol is injected in a triethylamine background. The behavior of $\mathbf{H}_{2} \mathbf{T P P}$ is more surprising. Here, when triethylamine is added to an ethanol background, resistance increases, decreasing only when ethanol is removed. However, the addition of ethanol to triethylamine elicits the expected increase in resistance.

The different behavior of the four sensors suggests that the balance of interactions in the same is rather different. In corrole-based sensors both the basic and donor character of the amine is the dominant feature: in the case of free-base corrole the amine leads to the anion formation, while in the case of the copper complex it induces the macrocyle reduction. These peculiar interactions for corrole led to the amine sensitivity, which prevails when the sensor is previously exposed to ethanol, and in presence of triethylamine the sensitivity to ethanol is disregarded. On the other hand, in porphyrins, the electron donor property of the amine seems less prevalent. Indeed, in CuTPP the intensity of coordination and other interactions are comparable, namely the sensor is sensitive to triethylamine in an atmosphere of ethanol and sensitive to ethanol in a triethylamine background. The case of $\mathbf{H}_{2}$ TPP is different. Here the binding of ethanol to the hydrogen atoms of the macrocycle core prevents any charge transfer between triethylamine and porphyrins; thus, the absorption of triethylamine can only increase the resistance. The removal of ethanol once again enables the charge transfer between triethylamine and porphyrin and in the second part of the experiment $\mathbf{H}_{2} \mathbf{T P P}$ and CuTPP follow the same behavior.

\section{Multivariate analysis and spherical PCA}

The different selectivity patterns make these sensors eligible as elements of a sensor array. A simple method to evaluate the properties of the sensor array is provided by Principal Component Analysis (PCA). Multivariate signals are naturally represented in a vector space where each sensor defines an orthogonal axis. The lack of selectivity means that the same compounds are sensed by more sensors, and sensor data are correlated. PCA is a simple and efficient algorithm to remove the correlation, defining a new orthogonal basis of uncorrelated variables. These variables are called principal components and they are obtained by a linear combination of sensor data.

Figure 6A shows the plot of the first two principal components of the PCA calculated with the responses of sensors to all the test compounds at variable concentrations (data shown in Figure S4 in Supporting Information). The data of each sensor are standardized (zero mean and unitary 
variance) before PCA is calculated. The total variance explained in the plot is about $98 \%$ of the total variance of the data. Scores emerge from the origin and, for each volatile compound, they are ordered in a sequence that follows the increase of concentration.

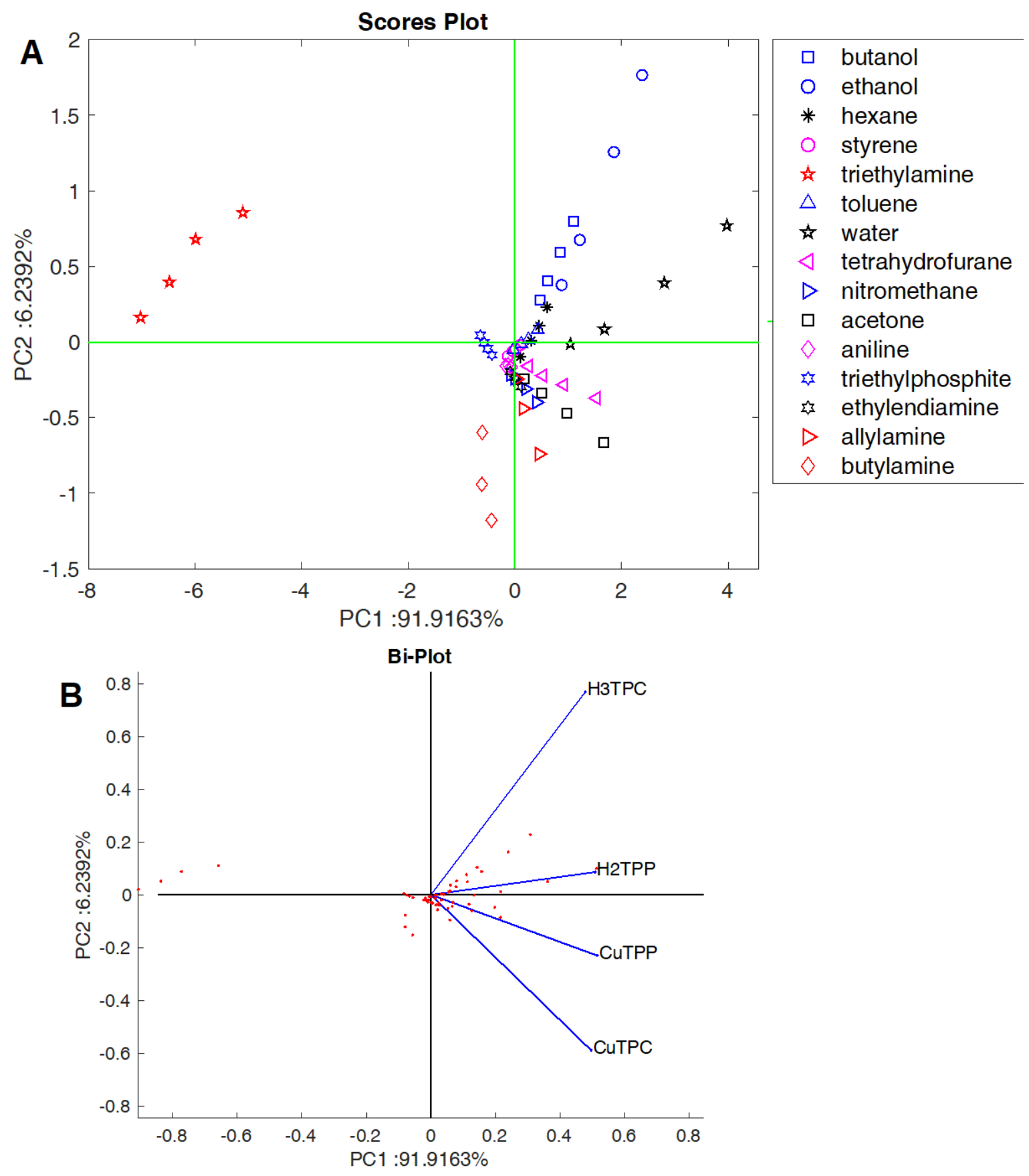

Figure 6: PCA of standardized sensors data. A) Scores plot of the first two principal components. Each symbol in the scores plot corresponds to a volatile compound and each data point to a different concentration. B) The corresponding bi-plot where loadings and scores are simultaneously plotted. 
The opposite sign of the response to triethylamine, butylamine and triethyl phosphite is clearly visible in the scores plot, as well polar compounds (alcohols and water) are also plotted in distinct regions. Figure 6B shows the bi-plot where loadings (the contribution of each sensor) are overlapped with scores. Since all sensors are proportional to the concentration, there is a very high correlation between sensors, and more than $91 \%$ of the total variance is carried by the first principal component. As a consequence, all sensor loadings are mostly aligned along the first principal component. It is interesting to note that corroles dominate the second principal component, with divergent contributions of $\mathbf{H}_{3}$ TPC and CuTPC. This behavior shows that the differences between $\mathbf{H}_{3}$ TPC and CuTPC are larger with respect to those of $\mathrm{H}_{2}$ TPP and CuTPP.

The variable concentration prevents the identification of volatile compounds. In other words, the multivariate data do not form close clusters for each compound. Cluster analysis applied to the standardized multivariate data shows that only triethylamine, butylamine and triethyl posphite data can be univocally identified (Figure S5 in the Supporting Information).

The identification of compounds can be greatly improved once the influence of the concentration in sensor data is removed. ${ }^{36}$ The responses of sensors are almost linear with the concentration (see Figure S4 in Supporting Information); thus, a simple normalization of the length of the multivariate data vector should be enough to cancel the dependence of the concentration. This is obtained dividing the signal of each sensor by the length of the corresponding vector:

$$
z_{i j}^{*}=\frac{z_{i j}}{\sqrt{\sum_{k} z_{k j}^{2}}}
$$

Where $\mathrm{z}_{\mathrm{ij}}$ is the response of the $\mathrm{i}$-th sensor to the $\mathrm{j}$-th compound and the sum is extended to all sensors of the array.

The response of a linear sensor is: $z_{i j}=S_{i j} \cdot c_{j}$. Where $\mathrm{S}_{\mathrm{ij}}$ is the sensitivity of the i-th sensor to the $\mathrm{j}$-th compound, and $c_{j}$ is the concentration. Since the concentration is common to all sensors, the normalization results in

$$
z_{i j}^{*}=\frac{S_{i j}}{\sqrt{\sum_{k} S_{k j}^{2}}}
$$


Namely, the normalized response of a sensor to a given compound corresponds to the sensitivity of the sensor normalized by the sum of all the sensitivities. The normalized data lie on the surface of a hypersphere of unitary radius.
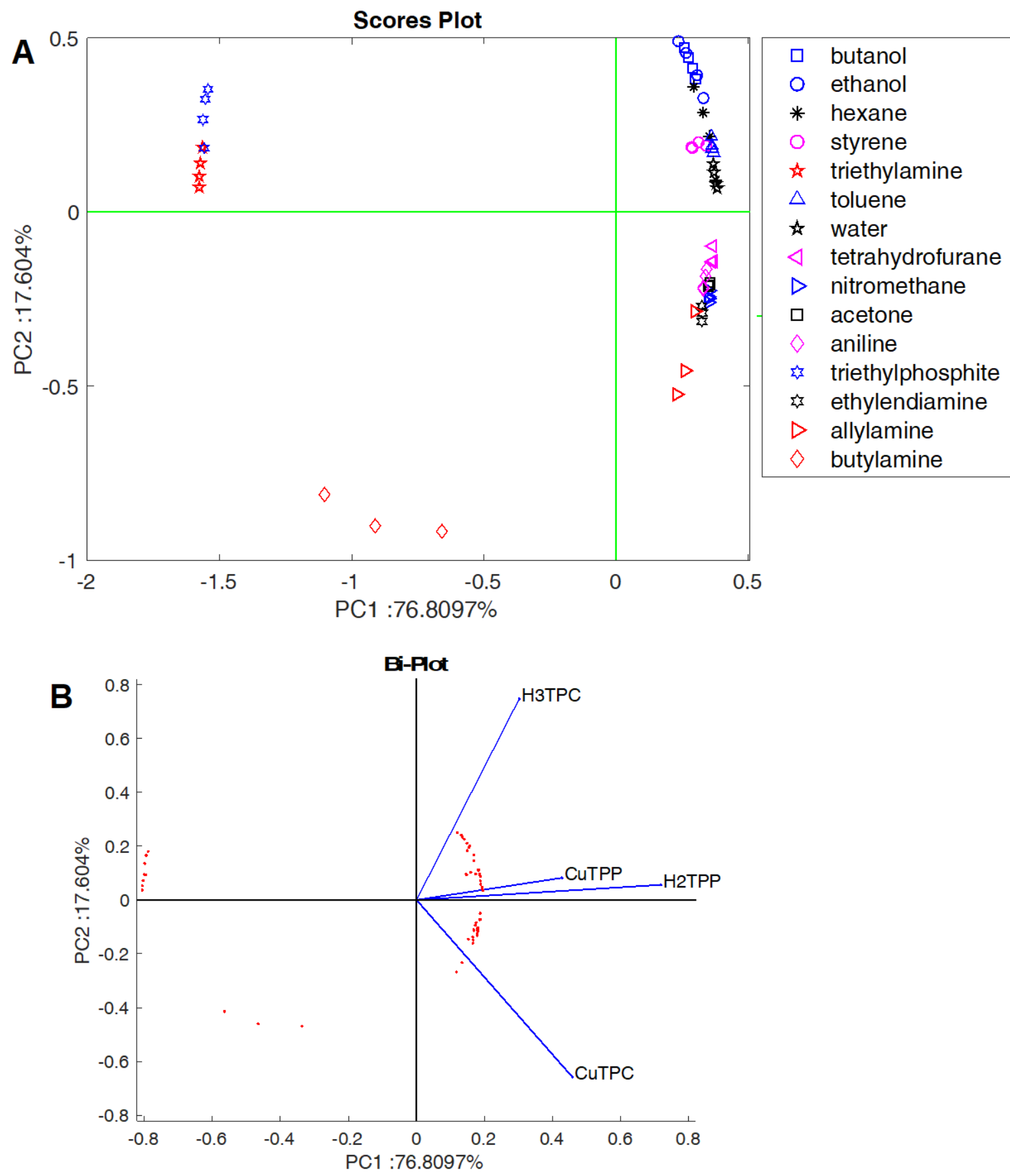

Figure 7: PCA of normalized sensors data. A) Scores plot of the first two principal components. $B)$ The Bi-plot shows both the loadings and the scores of the first two principal components.

Figure 7 shows the results of the PCA calculated on the normalized data. The removal of the concentration reduces the correlation among the sensors. The total variance explained by the 
first two principal components is still large (above 94\%) but it is more equally distributed between the principal components: about $77 \%$ and $17 \%$ in the first and second principal component, respectively.

Figure 7A shows the scores plot and Figure 7B the bi-plot. The loadings of the PCA of normalized data are qualitatively similar to those of the PCA calculated on the standardized data. The scores plot shows that the projection of the data in the subspace of the first two principal components preserves the hyper-spherical distribution of the data. However, the removal of the concentration is not enough to cluster each compound in a close region. This is partially due to the non-perfect linear dependence of sensor data on the concentration.

To correctly interpret this plot, we have to consider that data, which are separated on the hyperspherical surface may overlap when they are projected onto a plane. Thus, an appropriate analysis of normalized data should be based on spherical coordinates, namely on the angles that define the position of a point on the spherical surface. It is interesting to note that normalization reduces by one the dimensions of the data, e.g. only one angle is necessary to define the position on a bidimensional circle. A discussion about the comparison of linear and spherical principal component analysis is presented in the Supporting Information.

Recently, spherical principal component analysis was introduced as a method to reduce dimensions of data lying on a hypersphere where the angular distance is considered the equivalent of the usual Euclidean distance. ${ }^{27}$ Spherical principal components are calculated with an iterative minimization method. The algorithm easily converges to a stable solution, and the result depends on the initial conditions. However, repeated calculations with randomly chosen initial conditions provide very similar scores plots.

Figure 8 shows the scores plot of the first two spherical principal components of the normalized data. The differences with the "standard" PCA (shown in Figure 6) are rather evident. Data related to the same compound form well separated clusters, with the exception of the couples of ethanol and butanol and acetone and nitromethane whose data still appear overlapped in the plane of the first two spherical principal component. Cluster analysis applied to the whole set of four spherical principal components shows that nitromethane and acetone form separate cluster and thus their overlap in Figure 8 is a project effect (see Figure S7 in the Supporting Information). On the other hand cluster analysis confirms that ethanol and butanol cannot be distinguished one from the other. 


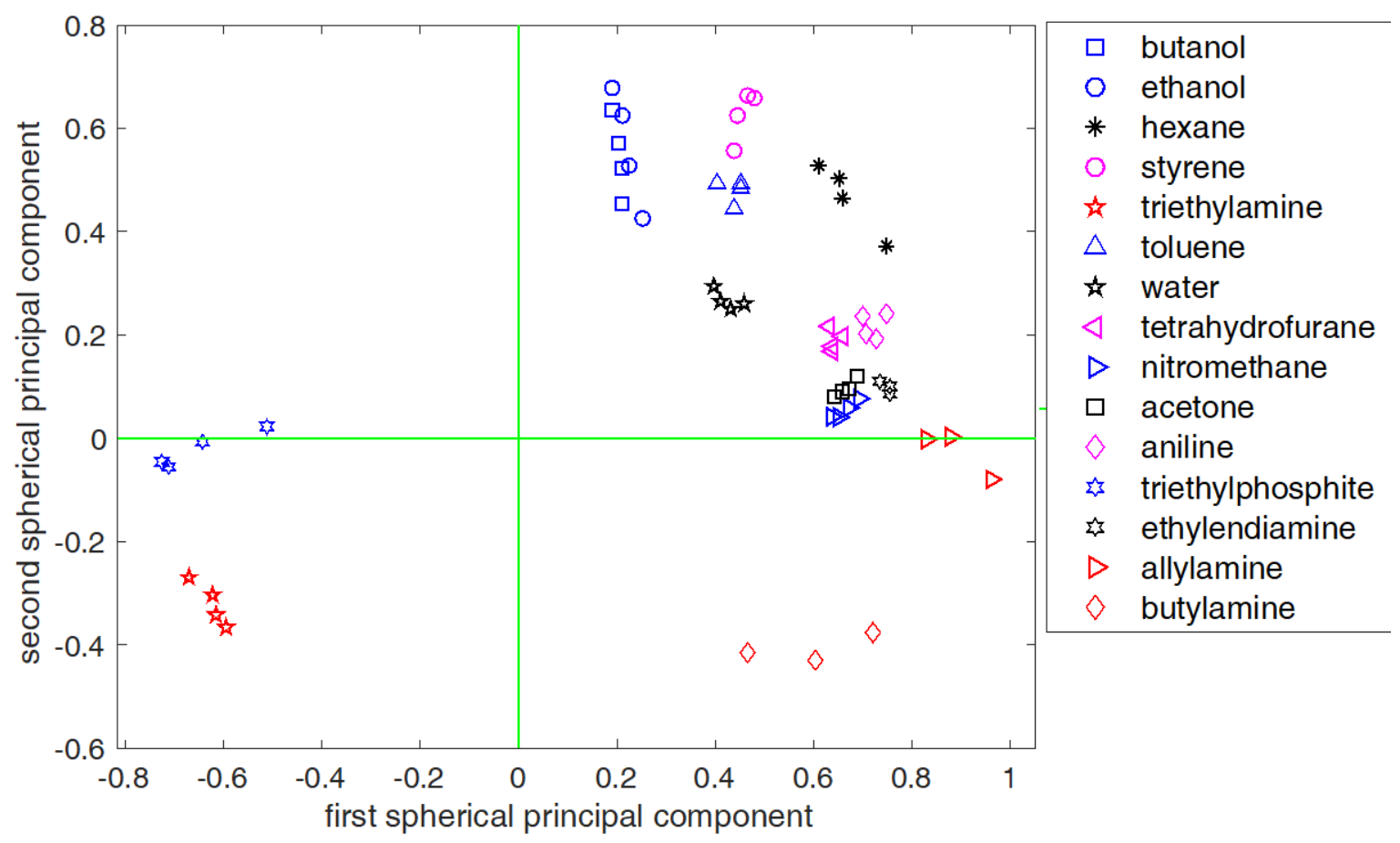

Figure 8: Plot of the first two spherical principal components.

\section{Chemotopic map}

The ordering of compounds in Figure 8 follows the chemical similarities between the investigated molecules. Thus, molecules with similar properties are plotted in proximity to each other.

However, the chemical families cover regions of different extension. For instance, a large part of the scores plot is occupied by amines, while other molecules such as nitromethane, acetone and tetrahydrofuran, although quite different from one another, lie in a restricted region. This uneven coverage of the scores plot indicates the preference of the array for the detection of amines.

The arrangement of compounds in Figure 8 resembles the chemotopic map of natural olfaction, where similar compounds activates close regions of the olfactory bulb. ${ }^{37} 38$ However, the chemotopic map in the olfactory bulb derives from the connection of receptor neurons to glomeruli, ${ }^{39}$ while in an electronic nose this map is not unique but it depends on the algorithm used. This similarity suggests using Figure 8 as a reference map to infer the chemical composition of unknown samples. 
To test this idea, real samples of supposedly known composition were measured. The samples were of sliced, and minced beef. They were measured after different storage times in a refrigerator.

Figure 9 shows he real sample data projected onto the first two spherical principal components plane. The meat data move, at different rates, towards the region of the amines. Sliced meat data show a slow evolution, while minced meat data immediately converge to the amines region indicating a saturation of amines production. This simple experiment shows that the map of compounds can be used as a sort of atlas, where the composition of an unknown sample can be inferred from its relative position respect to a set of reference compounds.

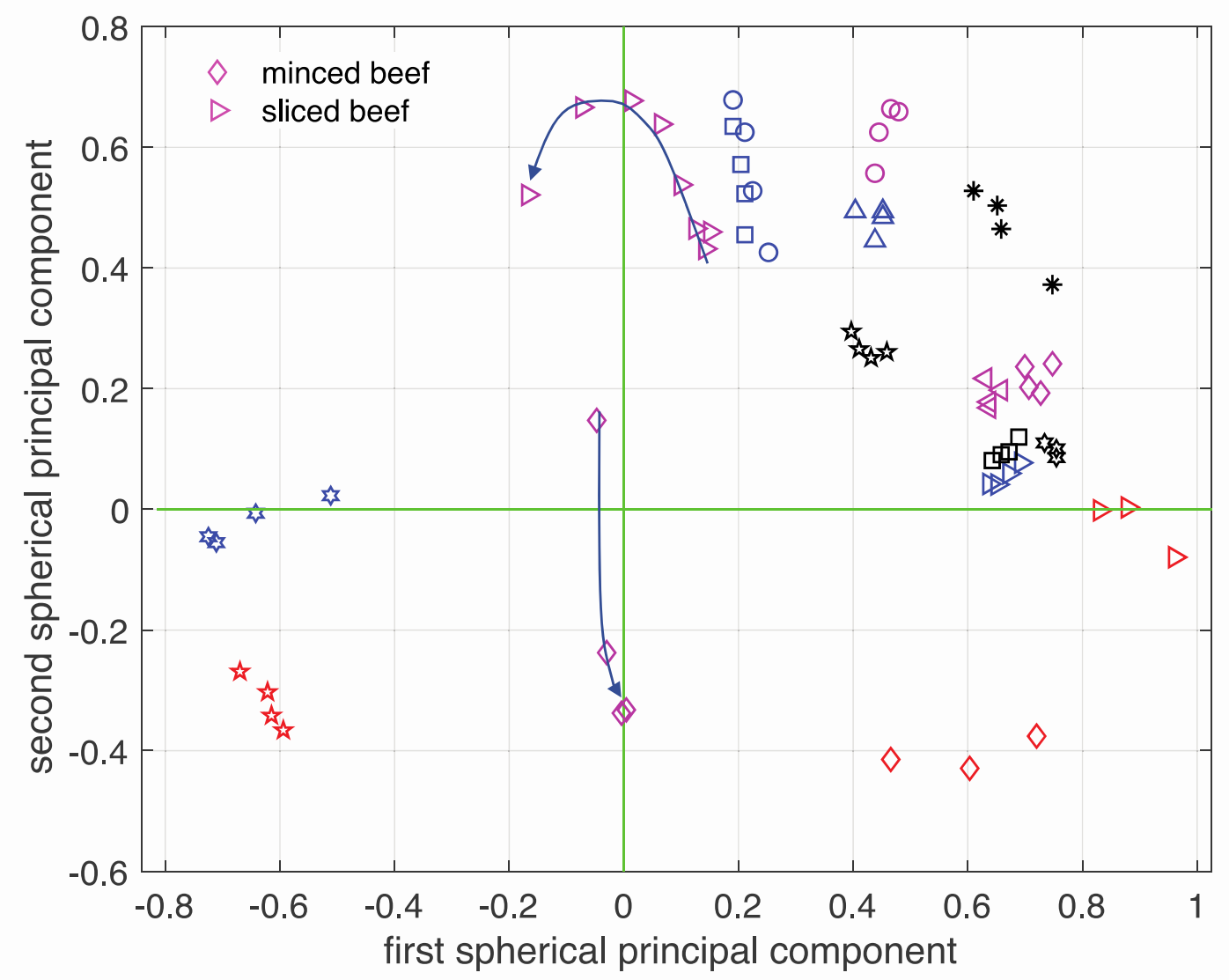

Figure 9: Projection of meat data in the plane of the first two spherical principal components. Arrows indicate the time progression in time of the meat spoilage process.

\section{Conclusions}

The photosensitivity of porphyrinoids coated $\mathrm{ZnO}$ nanoparticles activates the conductivity of the solid-layers of coated nanoparticles. The conductivity of these resistors is found to be modulated by the adsorption of volatile compounds, and the chemical sensitivity is driven by 
the properties of porphyrinoids. Sensors respond, even if with different intensity, to all the investigated volatile compounds, confirming that each porphyrinoids may host multiple interaction mechanisms. ${ }^{40}$ This property is well known, and has been fully exploited in quartz microbalance sensors, where arrays made of porphyrinoids with different molecular structures show a selectivity pattern sufficient for electronic nose applications in varied fields, such as medical diagnosis ${ }^{71}$ and microbial contamination. ${ }^{42}$.

Differently from quartz microbalances, where all interactions are equally considered, in porphyrinoid coated nanoparticles interactions with strong electron donors are highlighted, but without losing cross-selectivity. Thus, strong electron donor species (triethylamine, butylamine and triethyl phosphite, among those investigated) elicit a decrease of resistance, while exposure to the other investigated compounds results in an increase of resistance.

The measure of sensor response to triethylamine dispensed in a background of ethanol, and vice versa, enabled comparing different interaction mechanisms. Sensors behavior strongly depends on the functionalizing molecules. Corroles sensors show an absolute sensitivity to triethylamine with respect to ethanol, while in porphyrin coated sensors the first absorbed compound excludes the response to additional species. The dominance of charge transfers in corrole species can be explained on the basis of their peculiar structure. ${ }^{43}$ The largest reactivity of corrole also affects the nanoparticles morphology. With respect to porphyrins, corroles coated nanoparticles are larger and the typical hexagonal structure of $\mathrm{ZnO}$ is no longer observed.

Metal-free and copper complex of corroles and porphyrins sufficiently differentiate the respective sensors to provide the array made of these devices with a combinatorial selectivity that is able to identify the fifteen tested compounds independently from their concentration. For the objective, the data were properly analyzed with the spherical PCA algorithm. This proved to be very efficient to display data of opposite signs introducing, not the distance, but the angular separation between data. The resulting scores plot displays an arrangement of compounds in agreement with the chemical similarities among compounds. However, the regions of the scores plot are not equally distributed and the sensors' preference for amines is shown by the large portion occupied by amine data. The scores plot also serves as a sort of atlas to interpret samples of unknown composition. This was studied projecting into the scores plot the sensor data related to the spoilage processes in beef. The progression of spoilage in meat moves the samples towards the amines region, evidencing the most important compound in the process. 
In conclusion, porphyrinoid capped $\mathrm{ZnO}$ nanoparticles are a viable material for the development of sensors for electronic nose applications. The rich diversity of corroles and porphyrins and the manifold of available metal complexes is the basis for the implementation of the combinatorial selectivity that is the fundamental character of biological olfaction and the basis of electronic noses.

\section{Supporting information}

Supporting includes preparation of porphyrin and corrole derivatives, schemes S1-S2, figures S1-S7, comparison between principal component analysis and spherical principal component analysis.

The supporting information is available free of charge on the ACS Publications website.

\section{Acknowledgments}

This research was partly funded by INAIL in the framework of the project BRIC2019-ID7 and by Project SUNSET MIUR Italy, PRIN2017 Grant No. 2017EKCS35_002

\section{References}

(1) Cowen, T.; Karim, K.; Piletsky, S. Computational Approaches in the Design of Synthetic Receptors - A Review. Anal. Chim. Acta 2016, 936, 62-74. https://doi.org/10.1016/j.aca.2016.07.027.

(2) Mu, B.; Zhang, J.; McNicholas, T. P.; Reuel, N. F.; Kruss, S.; Strano, M. S. Recent Advances in Molecular Recognition Based on Nanoengineered Platforms. Acc. Chem. Res. 2014, 47 (4), 979-988. https://doi.org/10.1021/ar400162w.

(3) Israelachvili J. Intermolecular and Surface Forces; Elsevier B.V., 2011.

(4) Chiu, S.; Tang, K. Towards a Chemiresistive Sensor-Integrated Electronic Nose: A Review. Sensors2 2013, 13, 14214-14247.

(5) Paolesse, R.; Nardis, S.; Monti, D.; Stefanelli, M.; Di Natale, C. Porphyrinoids for Chemical Sensor Applications. Chem. Rev. 2017, 117 (4). https://doi.org/10.1021/acs.chemrev.6b00361.

(6) Gasparri, R.; Santonico, M.; Valentini, C.; Sedda, G.; Borri, A.; Petrella, F.; Maisonneuve, P.; Pennazza, G.; D’Amico, A.; Di Natale, C.; Paolesse, R.; Spaggiari, L. Volatile Signature for the Early Diagnosis of Lung Cancer. J. Breath Res. 2016, 10 (1). https://doi.org/10.1088/1752-7155/10/1/016007. 
(7) Zetola, N. M.; Modongo, C.; Matsiri, O.; Tamuhla, T.; Mbongwe, B.; Matlhagela, K.; Sepako, E.; Catini, A.; Sirugo, G.; Martinelli, E.; Paolesse, R.; Di Natale, C. Diagnosis of Pulmonary Tuberculosis and Assessment of Treatment Response through Analyses of Volatile Compound Patterns in Exhaled Breath Samples. J. Infect. 2017, 74 (4), 367-376. https://doi.org/10.1016/j.jinf.2016.12.006.

(8) Qi, P.; Vermesh, O.; Grecu, M.; Javey, A.; Wang, Q.; Dai, H.; Peng, S.; Cho, K. J. Toward Large Arrays of Multiplex Functionalized Carbon Nanotube Sensors for Highly Sensitive and Selective Molecular Detection. Nano Lett. 2003, 3 (3), 347-351. https://doi.org/10.1021/nl034010k.

(9) Wang, B.; Haick, H. Effect of Chain Length on the Sensing of Volatile Organic Compounds by Means of Silicon Nanowires. ACS Appl. Mater. Interfaces 2013, 5 (12), 5748-5756. https://doi.org/10.1021/am401265z.

(10) Paska, Y.; Haick, H. Interactive Effect of Hysteresis and Surface Chemistry on Gated Silicon Nanowire Gas Sensors. ACS Appl. Mater. Interfaces 2012, 4 (5), 2604-2617. https://doi.org/10.1021/am300288z.

(11) Yuan, H.; Kang, B.-H.; Jeong, H.-M.; Kwon, H.-C.; Yeom, S.-H.; Lee, J.-S.; Kwon, D.-H.; Kang, S.-W. Room Temperature VOC Gas Detection Using a Gated Lateral BJT with an Assembled Solvatochromic Dye. Sensors Actuators, B Chem. 2013, 187, 288-294. https://doi.org/10.1016/j.snb.2012.11.029.

(12) Lonergan, M. C.; Severin, E. J.; Doleman, B. J.; Beaber, S. A.; Grubbs, R. H.; Lewis, N. S. Array-Based Vapor Sensing Using Chemically Sensitive, Carbon Black-Polymer Resistors. Chem. Mater. 1996, 8 (9), 2298-2312. https://doi.org/10.1021/cm960036j.

(13) Wohltjen, H.; Snow, A. W. Colloidal Metal-Insulator-Metal Ensemble Chemiresistor Sensor. Anal. Chem. 1998, 70 (14), 2856-2859. https://doi.org/10.1021/ac9713464.

(14) Haick, H. Chemical Sensors Based on Molecularly Modified Metallic Nanoparticles. J. Phys. D. Appl. Phys. 2007, 40 (23), 7173-7186. https://doi.org/10.1088/00223727/40/23/S01.

(15) Cho, B.; Lee, K.; Pyo, S.; Kim, J. Fabrication and Characterization of VOC Sensor Array Based on $\mathrm{SnO}<\mathrm{inf}>2</ \mathrm{Inf}>$ and $\mathrm{ZnO}$ Nanoparticles Functionalized by Metalloporphyrins. Micro Nano Syst. Lett. 2018, 6 (1). https://doi.org/10.1186/s40486018-0072-3.

(16) Zhou, Y.; Gao, C.; Guo, Y. UV Assisted Ultrasensitive Trace NO $<$ inf $>2</$ Inf $>$ Gas Sensing Based on Few-Layer MoS <inf $>2</$ Inf $>$ Nanosheet-ZnO Nanowire Heterojunctions at Room Temperature. J. Mater. Chem. A 2018, 6 (22), 10286-10296. 
https://doi.org/10.1039/c8ta02679c.

(17) Comini, E.; Faglia, G.; Sberveglieri, G. UV Light Activation of Tin Oxide Thin Films for $\mathrm{NO}<\mathrm{inf}>2</ \mathrm{Inf}>$ Sensing at Low Temperatures. Sensors Actuators, B Chem. 2001, 78 (1-3), 73-77. https://doi.org/10.1016/S0925-4005(01)00796-1.

(18) Robertson, N. Optimizing Dyes for Dye-Sensitized Solar Cells. Angew. Chemie - Int. Ed. 2006, 45 (15), 2338-2345. https://doi.org/10.1002/anie.200503083.

(19) Campbell, W. M.; Burrell, A. K.; Officer, D. L.; Jolley, K. W. Porphyrins as Light Harvesters in the Dye-Sensitised $\mathrm{TiO}<\mathrm{inf}>2</ \mathrm{Inf}>$ Solar Cell. Coord. Chem. Rev. 2004, 248 (13-14), 1363-1379. https://doi.org/10.1016/j.ccr.2004.01.007.

(20) Sivalingam, Y.; Martinelli, E.; Catini, A.; Magna, G.; Pomarico, G.; Basoli, F.; Paolesse, R.; Di Natale, C. Gas-Sensitive Photoconductivity of PorphyrinFunctionalized ZnO Nanorods. J. Phys. Chem. C 2012, 116 (16), 9151-9157. https://doi.org/10.1021/jp302225u.

(21) Magna, G.; Catini, A.; Kumar, R.; Palmacci, M.; Martinelli, E.; Paolesse, R.; di Natale, C. Conductive Photo-Activated Porphyrin-ZnO Nanostructured Gas Sensor Array. Sensors (Switzerland) 2017, 17 (4). https://doi.org/10.3390/s17040747.

(22) Nardis, S.; Monti, D.; Paolesse, R. Novel Aspects of Corrole Chemistry. Mini. Rev. Org. Chem. 2005, 2 (4), 355-374. https://doi.org/10.2174/157019305774322716.

(23) Nardis, S.; Mandoj, F.; Stefanelli, M.; Paolesse, R. Metal Complexes of Corrole. Coord. Chem. Rev. 2019, 388, 360-405. https://doi.org/10.1016/j.ccr.2019.02.034.

(24) Zhang, W.; Lai, W.; Cao, R. Energy-Related Small Molecule Activation Reactions: Oxygen Reduction and Hydrogen and Oxygen Evolution Reactions Catalyzed by Porphyrin- and Corrole-Based Systems. Chem. Rev. 2017, 117 (4), 3717-3797. https://doi.org/10.1021/acs.chemrev.6b00299.

(25) Teo, R. D.; Hwang, J. Y.; Termini, J.; Gross, Z.; Gray, H. B. Fighting Cancer with Corroles. Chem. Rev. 2017, 117 (4), 2711-2729. https://doi.org/10.1021/acs.chemrev.6b00400.

(26) Agresti, A.; Berionni Berna, B.; Pescetelli, S.; Catini, A.; Menchini, F.; Di Natale, C.; Paolesse, R.; Di Carlo, A. Copper-Based Corrole as Thermally Stable Hole Transporting Material for Perovskite Photovoltaics. Adv. Funct. Mater. 2020. https://doi.org/10.1002/adfm.202003790.

(27) Liu, K.; Li, Q.; Wang, H.; Tang, G. Spherical Principal Component Analysis. In SIAM International Conference on Data Mining, SDM 2019; 2019; pp 387-395. https://doi.org/10.1137/1.9781611975673.44. 
(28) Stefanelli, M.; Monti, D.; Van Axel Castelli, V.; Ercolani, G.; Venanzi, M.; Pomarico, G.; Paolesse, R. Chiral Supramolecular Capsule by Ligand Promoted Self-Assembly of Resorcinarene-Zn Porphyrin Conjugate. J. Porphyr. Phthalocyanines 2008, 12 (12), 1279-1288. https://doi.org/10.1142/S1088424608000662.

(29) Stefanelli, M.; Monti, D.; Venanzi, M.; Paolesse, R. Kinetic and Spectroscopic Studies on the Self-Aggregation of a Meso-Substituted Amphiphilic Corrole Derivative. New J. Chem. 2007, 31 (10), 1722-1725. https://doi.org/10.1039/b709723a.

(30) Magna, G.; Zor, S.; Catini, A.; Capuano, R.; Basoli, F.; Martinelli, E.; Paolesse, R.; Di Natale, C. Surface Arrangement Dependent Selectivity of Porphyrins Gas Sensors. Sensors Actuators B. Chem. 2017, 251, 524-532.

(31) Rochford, J.; Chu, D.; Hagfeldt, A.; Galoppini, E. Tetrachelate Porphyrin Chromophores for Metal Oxide Semiconductor Sensitization: Effect of the Spacer Length and Anchoring Group Position. J. Am. Chem. Soc. 2007, 129 (15), 4655-4665. https://doi.org/10.1021/ja068218u.

(32) Yahiro, J.; Kawano, T.; Imai, H. Nanometric Morphological Variation of Zinc Oxide Crystals Using Organic Molecules with Carboxy and Sulfonic Groups. J. Colloid Interface Sci. 2007, 310 (1), 302-311. https://doi.org/10.1016/j.jcis.2007.01.072.

(33) Wang, L.; Muhammed, M. Synthesis of Zinc Oxide Nanoparticles with Controlled Morphology. J. Mater. Chem. 1999, 9 (11), 2871-2878.

https://doi.org/10.1039/a907098b.

(34) D’Amico, A.; Di Natale, C. A Contribution on Some Basic Definitions of Sensors Properties. IEEE Sens. J. 2001, 1 (3), 183-190.

https://doi.org/10.1109/JSEN.2001.954831.

(35) Ding, T.; Alemán, E. A.; Modarelli, D. A.; Ziegler, C. J. Photophysical Properties of a Series of Free-Base Corroles. J. Phys. Chem. A 2005, 109 (33), 7411-7417. https://doi.org/10.1021/jp052047i.

(36) Horner, G.; Hierold, C. Gas Analysis by Partial Model Building. Sensors Actuators B. Chem. 1990, 2 (3), 173-184. https://doi.org/10.1016/0925-4005(90)85002-G.

(37) Mori, K.; Takahashi, Y. K.; Igarashi, K. M.; Yamaguchi, M. Maps of Odorant Molecular Features in the Mammalian Olfactory Bulb. Physiol. Rev. 2006, 86 (2), 409-433. https://doi.org/10.1152/physrev.00021.2005.

(38) Johnson, B. A.; Leon, M. Chemotopic Odorant Coding in a Mammalian Olfactory System. J. Comp. Neurol. 2007, 503 (1), 1-34. https://doi.org/10.1002/cne.21396.

(39) Manzini, I.; Korsching, S. The Peripheral Olfactory System of Vertebrates: Molecular, 
Structural and Functional Basics of the Sense of Smell | Das Periphere Olfaktorische System von Vertebraten: Molekulare, Strukturelle Und Funktionelle Grundlagen Des Riechens. Neuroforum 2011, 17 (3), 110-118.

(40) Di Natale, C.; Monti, D.; Paolesse, R. Chemical Sensitivity of Porphyrin Assemblies. Mater. Today 2010, 13 (7-8). https://doi.org/10.1016/S1369-7021(10)70127-9.

(41) Capuano, R.; Spitalieri, P.; Talarico, R. V.; Catini, A.; Domakoski, A. C.; Martinelli, E.; Scioli, M. G.; Orlandi, A.; Cicconi, R.; Paolesse, R.; Di Natale, C.; Sangiuolo, F. Volatile Compounds Emission from Teratogenic Human Pluripotent Stem Cells Observed during Their Differentiation in Vivo. Sci. Rep. 2018, 8 (1). https://doi.org/10.1038/s41598-018-29212-0.

(42) Capuano, R.; Paba, E.; Mansi, A.; Marcelloni, A. M.; Chiominto, A.; Proietto, A. R.; Zampetti, E.; Macagnano, A.; Lvova, L.; Catini, A.; Tranfo, G.; Di Natale, C. Aspergillus Species Discrimination Using a Gas Sensor Array. Sensors (Switzerland) 2020, 20 (14), 1-12. https://doi.org/10.3390/s20144004.

(43) Ghosh, A. Electronic Structure of Corrole Derivatives: Insights from Molecular Structures, Spectroscopy, Electrochemistry, and Quantum Chemical Calculations. Chem. Rev. 2017, 117 (4), 3798-3881. https://doi.org/10.1021/acs.chemrev.6b00590. 